\title{
Epigallocatechin-3-gallate ameliorates insulin resistance in hepatocytes
}

\author{
SHAN-BO MA ${ }^{1 *}$, RUI ZHANG ${ }^{2 *}$, SHAN MIAO $^{3 *}$, BIN GAO $^{4 *}$, YANG LU $^{1}$, \\ SEN HUI ${ }^{1}$, LONG LI $^{1}$, XIAO-PENG SHI ${ }^{1}$ and AI-DONG WEN ${ }^{1}$
}

\begin{abstract}
Departments of ${ }^{1}$ Pharmacy and ${ }^{2}$ Otolaryngology-Head and Neck Surgery, Xijing Hospital, ${ }^{3}$ Institute of Medical Materials, Fourth Military Medical University; ${ }^{4}$ Department of Endocrinology, Xijing Hospital, Fourth Military Medical University, Xi'an, Shaanxi 710032, P.R. China
\end{abstract}

Received December 13, 2015; Accepted December 19, 2016

DOI: $10.3892 / \mathrm{mmr} .2017 .6450$

\begin{abstract}
Hyperglycemia is a typical pathogenic factor in a series of complications among patients with type II diabetes. Epigallocatechin-3-gallate (EGCG) is the major polyphenol extracted from green tea and is reported to be an antioxidant. The aim of the present study was to examine the effect of EGCG on insulin resistance in human HepG2 cells pretreated with high concentrations of glucose. The protein kinase B (AKT)/glycogen synthase kinase (GSK) pathways were analyzed using western blot analysis in HepG2 cells and primary mouse hepatocytes treated with high glucose and/or EGCG. Cellular glycogen content was determined using a glycogen assay kit. Reactive oxygen species (ROS) production was determined using dihydroethidium staining and flow cytometry. c-JUN N-terminal kinase (JNK)/insulin receptor substrate 1 (IRS1)/AKT/GSK signaling was explored using western blot analysis in HepG2 cells treated with high glucose and/or EGCG or N-acetylcysteine. High glucose significantly decreased the levels of phosphorylated AKT and GSK in HepG2 cells and mouse primary hepatocytes. Pretreatment with EGCG significantly restored the activation of AKT and GSK in HepG2 cells and primary hepatocytes exposed to high glucose. In HepG2 cells and primary hepatocytes, glycogen synthesis was improved by EGCG treatment in a dose-dependent manner. High glucose significantly stimulated the production of ROS while EGCG protected high glucose-induced ROS production. ROS is known
\end{abstract}

Correspondence to: Professor Xiao-Peng Shi or Professor Ai-Dong Wen, Department of Pharmacy, Xijing Hospital, Fourth Military Medical University, 127 Chang Le West Road, Xi'an, Shaanxi 710032, P.R. China

E-mail: shixiaop@fmmu.edu.cn

E-mail: adwen-2004@hotmail.com

*Contributed equally

Key words: epigallocatechin-3-gallate, reactive oxygen species, c-JUN N-terminal kinase/insulin receptor substrate 1/protein kinase B/ glycogen synthase kinase signaling pathway, insulin resistance to serve a major role in high glucose induced-insulin resistance by increasing JNK and IRS1 serine phosphorylation. In the present study, EGCG was observed to enhance the insulin-signaling pathway. EGCG ameliorated high glucose-induced insulin resistance in the hepatocytes by potentially decreasing ROS-induced JNK/IRS1/AKT/GSK signaling.

\section{Introduction}

Metabolic syndrome refers to a subset of metabolic abnormalities, which may increase the likelihood of developing cardiovascular disease and type II diabetes (1). In recent years, metabolic syndrome has become an increasing public health concern. It is estimated that 250 million people will suffer from type II diabetes by the year 2020 (2). Therefore, determining appropriate treatment strategies is particularly important for public health. Currently, the specific factors that lead to type II diabetes remain unknown. However, it is clear that insulin resistance serves a major role in the development of type II diabetes (3). Metabolic syndrome is characterized by insulin resistance, which is defined as impaired insulin function in target organs (4). The liver is an important insulin-sensitive organ, particularly in the regulation of glucose homeostasis. Hepatic insulin resistance is believed to induce a series of systematic consequences, and clinical observations have confirmed that insulin resistance is closely associated with hepatic pathologies (5).

Tea is a popular beverage consumed worldwide. Compared with other types of tea, green tea has been reported to exert various beneficial effects on metabolic syndrome (6). For instance, green tea consumption has been demonstrated to improve fat oxidation, which prevents obesity and insulin resistance in healthy individuals (7). In a previous clinical study, frequent consumption of green tea was associated with a decreased risk of type II diabetes, and it improved metabolic syndrome by decreasing insulin resistance and increasing glucose tolerance (6).

Polyphenolic compounds extracted from green tea have been demonstrated to increase insulin sensitivity (8-11). Among these polyphenolic compounds, epigallocatechin-3-gallate (EGCG) reduces the fecal lipid content and prompts fecal cholesterol and fat excretion in rats fed on a high fat diet (12). In addition, a considerable number of studies have demonstrated that tea polyphenols decrease obesity and hyperlipidemia, primarily 
by inhibiting lipolysis and enhancing fat emulsification $(13,14)$. However, a limited number of studies have investigated the mechanisms associated with green tea consumption and glucose metabolism in insulin-sensitive organs.

In order to investigate the specific effects of tea polyphenols on insulin resistance, the present study examined the effect of EGCG on insulin resistance in human HepG2 cells exposed to high glucose.

\section{Materials and methods}

EGCG. EGCG was purchased from Shanghai SolarBio Bioscience and Technology Co., Ltd. (Shanghai, China). To determine the dose-response association of EGCG on glycogen synthesis and lipogenesis, a series of molar concentrations $(0.01$, $0.1,1.0$ and $10 \mu \mathrm{M})$ of EGCG were prepared as previously reported (15).

Cell culture and treatments. The human hepatocyte cell line HepG2 (ref. no. ATCCHB8065; American Type Culture Collection, Manassas, VA, USA) were maintained in Dulbecco's modified Eagle's medium (DMEM; Gibco; Thermo Fisher Scientific, Inc., Waltham, MA, USA) containing normal glucose (5 mM), supplemented with $10 \%$ fetal bovine serum (FBS; Thermo Fisher Scientific, Inc.) and $100 \mathrm{U} / \mathrm{ml}$ penicillin (Thermo Fisher Scientific, Inc.), in an incubator at $37^{\circ} \mathrm{C}$ and $5 \% \mathrm{CO}_{2}$. HepG2 cells were cultured in complete media (CM) with $10 \%$ FBS until $70 \%$ confluence was reached. At $24 \mathrm{~h}$ prior to all experimental procedures, 5 or $30 \mathrm{mM}$ D-glucose (Thermo Fisher Scientific, Inc.), termed low glucose (LG) and high glucose (HG) respectively, were added to cells.

Cells $\left(4 \times 10^{3}\right)$ were seeded on 24-well plates for all assays. When the cells reached $90 \%$ confluence, the CM was discarded and starvation medium (SM) containing $0.5 \%$ FBS was added. Following incubation at $37^{\circ} \mathrm{C}$ with SM for $6 \mathrm{~h}, 100 \mathrm{nM}$ insulin (Eli Lilly Australia Pty, Ltd., Melrose Park, NSW, Australia) was added to each well, followed by the addition of $0.01-10 \mu \mathrm{M}$ EGCG to the appropriate wells in duplicate. Plates were then maintained for $24 \mathrm{~h}$ in $5 \% \mathrm{CO}_{2}$ at $37^{\circ} \mathrm{C}$. This treatment procedure was used for the purposes of all experiments in the present study.

Isolation of mouse primary hepatocytes. A single male C57BL/6J mouse (weight, 20-25 g; age, 12 weeks) was obtained from the Peking University Health Science Center (Beijing, China). It was maintained in a constructed shelter at $20-26^{\circ} \mathrm{C}, 30-60 \%$ humidity, 12:12 light/dark cycle, and provided with adequate supplies of food and fresh water. All experiments were approved by the Institutional Animal Care and Use Committee at the Fourth Military Medical University (Xi'an, China; no. 20150302). The liver was removed and primary hepatocytes were isolated using a two-step collagenase perfusion method $(0.2 \mathrm{mg} / \mathrm{ml}$ type IV collagenase in Hanks' balanced salt solution; Sigma-Aldrich; Merck Millipore, Darmstadt, Germany) as described previously (15). The hepatocytes were collected by centrifugation at $100 \mathrm{x} \mathrm{g}$ for $8 \mathrm{~min}$ at room temperature. The cells were immediately re-suspended in pre-warmed William's E medium (Merck Millipore) supplemented with 10\% FBS, $20 \mathrm{ng} / \mathrm{ml}$ dexamethasone (Merck Millipore), an insulin (5 mg/l)-transferrin $(5 \mathrm{mg} / \mathrm{l})$-sodium selenite $(5 \mathrm{~g} / \mathrm{l})$ solution (Merck Millipore), and $10 \mathrm{~g} / \mathrm{ml}$ gentamicin (Invitrogen; Thermo Fisher Scientific, Inc.).
The hepatocytes were then plated in collagen-coated $25 \mathrm{~cm}^{2}$ flasks at $1 \times 10^{6}$ cells/flask.

Measurement of glycogen content. Following pretreatment with 5 and $30 \mathrm{mM}$ D-glucose, glycogen levels were measured in the HepG2 cells and primary hepatocytes at $37^{\circ} \mathrm{C}$ for $3 \mathrm{~h}$ in the presence of $100 \mathrm{nmol} / \mathrm{l}$ insulin (US Biological, Salem, MA, USA), using a Glycogen Assay kit (BioVision, Inc., Milpitas, CA, USA), according to the manufacturer's protocol. Briefly, for hydrolysis, the Enzyme Mixture was added and the plates were incubated for $30 \mathrm{~min}$ at $37^{\circ} \mathrm{C}$; then the Glycogen Fluorometric Detector solution, which contains 10-acetyl-3,7-dihydroxyphenoxazine, was added and the fluorescence signal was monitored with an excitation wavelength of 530-540 $\mathrm{nm}$ and an emission wavelength of $585-595 \mathrm{~nm}$.

Western blotting. Cellular proteins were extracted using radioimmunoprecipitation assay buffer [50 mM Tris/HCl, $\mathrm{pH} 7.4$; 150 mM NaCl; $1 \%$ (v/v) nonidet P-40; $0.1 \%$ (w/v) SDS; Shanghai SolarBio Bioscience and Technology, Co., Ltd.] containing $1 \%$ (v/v) phenylmethanesulfonyl fluoride (Shanghai SolarBio Bioscience and Technology, Co., Ltd.), 0.3\% (v/v) protease inhibitor (Merck Millipore) and 0.1\% (v/v) phosphorylated proteinase inhibitor (Merck Millipore). Lysates were centrifuged at $1,000 \times \mathrm{g}$ and $4^{\circ} \mathrm{C}$ for $15 \mathrm{~min}$, and the supernatant was collected to obtain total protein. A Bicinchoninic Acid Protein assay kit (Pierce; Thermo Fisher Scientific, Inc.) was used to determine the protein concentration. Equal amounts of protein $(15 \mu \mathrm{g})$ were loaded onto a SDS-PAGE gel [10\% (v/v) polyacrylamide] and transferred onto a polyvinylidene difluoride membrane. Nonspecific binding was blocked using $8 \%(\mathrm{w} / \mathrm{v})$ milk in TBST (5\% Tween-20) for $2 \mathrm{~h}$ at room temperature. The membranes were then incubated with primary antibodies against phosphorylated (p)-insulin receptor substrate 1 (IRS1, Ser307; ab1194; 1:250), IRS1 (ab52167; 1:500), p-glycogen synthase kinase (p-GSK3 $\beta$, Ser9; ab131097; 1:500), GSK3 $\beta$ (ab170191; 1:1,000), p-protein kinase B (p-AKT; ab8933; 1:500), AKT (ab64148; 1:1,000) (Abcam, Shanghai, China) and $\beta$-actin (sc130300; 1:1,000; Santa Cruz Biotechnology, Inc., Dallas, TX, USA) overnight at $4^{\circ} \mathrm{C}$. Following several washes with TBST, the membranes were incubated in horseradish peroxidase (HRP)-conjugated goat anti-rabbit (ab97051) or goat anti-mouse immunoglobulin G (ab6789; Abcam) at a 1:5,000 dilution for $2 \mathrm{~h}$ at room temperature and then washed with phosphate-buffered saline (PBS). The target proteins were visualized using enhanced chemiluminescence (Merck Millipore) according to the manufacturer's recommendations, quantified using densitometry analysis and normalized against $\beta$-actin. Data are expressed as the fold-change compared to the $\beta$-actin.

To further determine whether the EGCG-associated enhancement of insulin signaling was due to scavenging reactive oxygen species (ROSs), the antioxidant $\mathrm{N}$-acetyl-cysteine (NAC; $10 \mathrm{mM}$; Beyotime Institute of Biotechnology, Jiangsu, China) was added either with or without EGCG, and the cells were incubated for $1 \mathrm{~h}$. Then the whole proteins were extracted and western blotting was performed as aforementioned.

Determination of ROS. HepG2 cells cultured on 6-well chamber slides $\left(1 \times 10^{6}\right)$ were washed with PBS three times for $5 \mathrm{~min} /$ wash, and the slides were incubated with the ROS fluorescent probe 

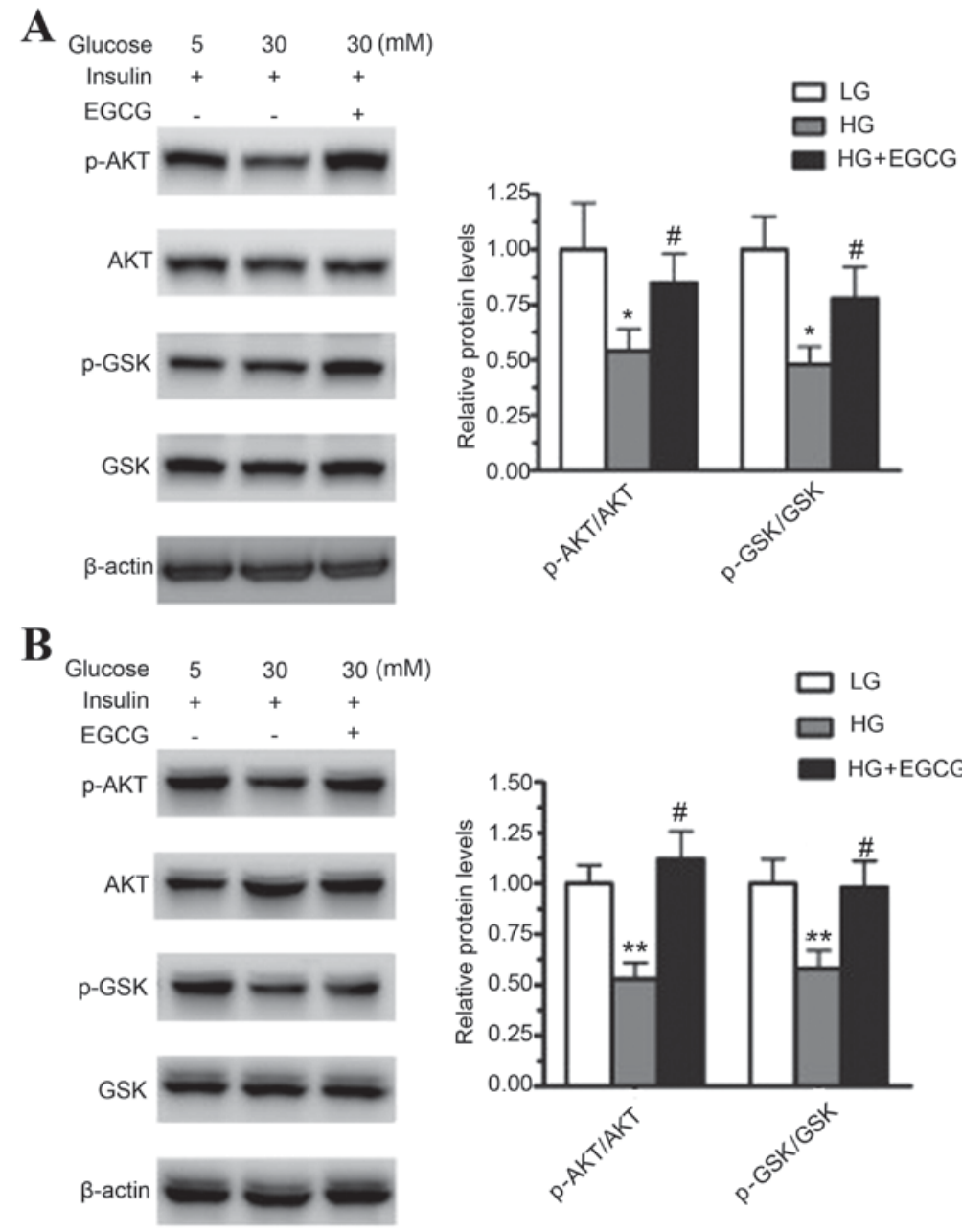

Figure 1. Effect of EGCG on the expression of p-AKT/AKT and p-GSK/GSK in (A) HepG2 cells and (B) primary hepatocytes. $\beta$-actin was used as a loading control. Representative western blots of AKT and GSK3 $\beta$ phosphorylation from three independent experiments are depicted. Quantitative data are expressed as the mean \pm standard deviation. " $\mathrm{P}<0.05$ and ${ }^{* *} \mathrm{P}<0.01$ vs. LG; ${ }^{*} \mathrm{P}<0.05$ vs. HG. EGCG, epigallocatechin-3-gallate; p, phosphorylated; GSK, glycogen synthase kinase; AKT, protein kinase B; LG, low glucose; HG, high glucose.

dihydroethidium (DHE; Vigorous Biotechnology Beijing Co., Ltd., Beijing, China) in serum-free DMEM/F-12 medium (Thermo Fisher Scientific, Inc.) for $30 \mathrm{~min}$ at $37^{\circ} \mathrm{C}$ in the dark. The slides were then fixed in $4 \%$ paraformaldehyde for $30 \mathrm{~min}$ at room temperature. The slides were washed with PBS and mounted. Immunofluorescence images were captured by fluorescence microscopy.

ROS production. Intracellular ROS generation was monitored by flow cytometry using the peroxide-sensitive fluorescent probe, 2',7'-dichlorofluorescin diacetate (DCFH-DA; Molecular Probes; Thermo Fisher Scientific, Inc.) as described previously (16). DCFH-DA is converted by intracellular esterases to DCFH, which is oxidized to the highly fluorescent DCF in the presence of an oxidant.

HepG2 cells (50-60\% confluent) were pre-incubated with LG (5 mM D-glucose) or HG (30 mM D-glucose) for $24 \mathrm{~h}$ at $37^{\circ} \mathrm{C}$, then treated with $5 \mu \mathrm{M}$ EGCG. Finally, cells were washed twice with $1 \mathrm{X}$ PBS and diluted to $2-3 \times 10^{6}$ cells $/ \mathrm{ml}$ in $10 \mathrm{mmol} / 1 \mathrm{DCHF}-\mathrm{DA}$ dyes. Cells were then incubated at room temperature for $30 \mathrm{~min}$ in the dark. The cells were subsequently analyzed by flow cytometry (Becton Dickinson; BD Biosciences, Franklin Lakes, NJ, USA).
Statistical analysis. All experiments were repeated at least three times. Statistical analyses were performed using SPSS software, version 22.0 (IBM SPSS, Armonk, NY, USA). The data are expressed as the mean \pm standard deviation and were analyzed using one-way analysis of variance followed by the Tukey-Kramer multiple comparison test. $\mathrm{P}<0.05$ was considered to indicate a statistically significant difference.

\section{Results}

EGCG activates the AKT/GSK pathway in HepG2 cells and primary hepatocytes. To determine the effect of $\mathrm{HG}$ on the AKT/GSK signaling pathway, HepG2 cells and mouse primary hepatocytes were treated with 30 or $5 \mathrm{mM}$ glucose for $24 \mathrm{~h}$. HG treatment significantly decreased the protein expression levels of phosphorylated AKT and GSK when compared to that of the LG treatment in HepG2 cells (p-AKT, P $<0.05$; p-GSK, $\mathrm{P}<0.05)$ and mouse primary hepatocytes (p-AKT, $\mathrm{P}<0.01$; p-GSK, P<0.01; Fig. 1). The effect of EGCG on the levels of phosphorylated AKT and GSK in HG-treated HepG2 cells and primary hepatocytes was then analyzed. Pretreatment with EGCG significantly restored the levels of p-AKT and p-GSK in HepG2 cells ( $\mathrm{p}-\mathrm{AKT}, \mathrm{P}<0.05$; $\mathrm{p}-\mathrm{GSK}, \mathrm{P}<0.05$ ) and primary 

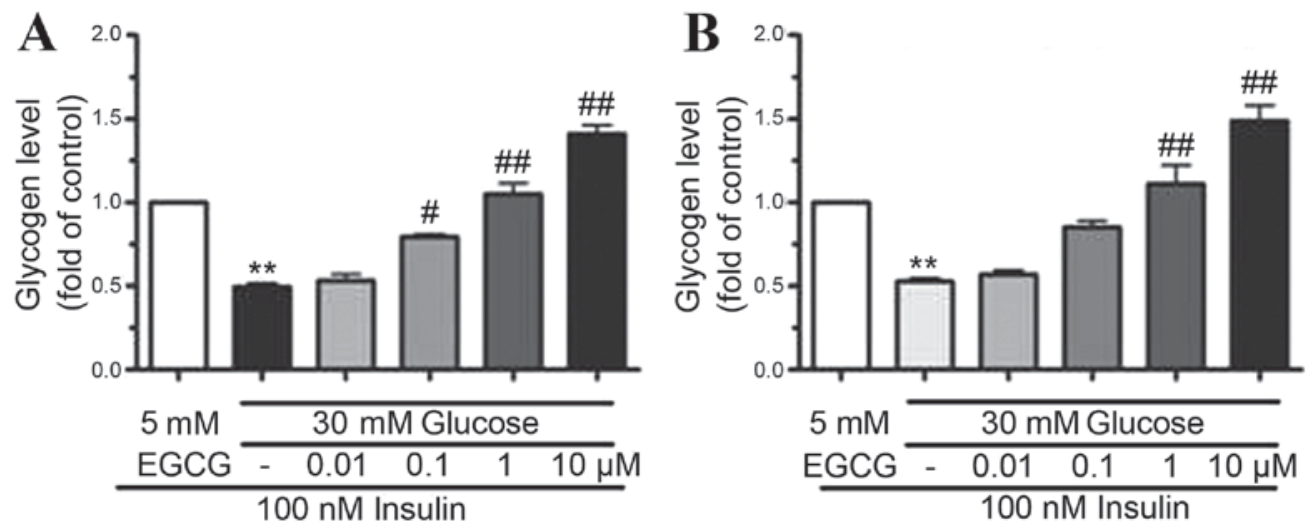

Figure 2. Glycogen synthesis in response to EGCG treatment in (A) HepG2 cells and (B) primary hepatocytes. The cells were pretreated with HG (30 mM) or LG $(5 \mathrm{mM})$ for $24 \mathrm{~h}$, prior to incubation with various concentrations of EGCG $(0.1-10 \mu \mathrm{M})$ plus $100 \mathrm{nM}$ insulin for a further $24 \mathrm{~h}$. HepG2 cells cultured with HG but without insulin were used to confirm that the HepG2 cells developed insulin resistance. Data are expressed as the mean \pm standard deviation of five individual experiments. ${ }^{* *} \mathrm{P}<0.01$ vs. $5 \mathrm{mM}$ glucose $+100 \mathrm{nM}$ insulin; ${ }^{\#} \mathrm{P}<0.05$ and ${ }^{\# \#} \mathrm{P}<0.01$ vs. $30 \mathrm{nM}$ glucose $+100 \mathrm{nM}$ insulin. EGCG, epigallocatechin-3-gallate; HG, high glucose; LG, low glucose.

hepatocytes ( $\mathrm{p}$-AKT, $\mathrm{P}<0.05$; $\mathrm{p}-\mathrm{GSK}, \mathrm{P}<0.05$ ) when compared to cells exposed to HG alone (Fig. 1). These results indicated that HG impaired insulin signaling in HepG2 cells and mouse primary hepatocytes, and EGCG improved HG-induced insulin resistance in the two cell types.

EGCG increased glycogen synthesis in HepG2 cells and primary hepatocytes. To determine the effect of EGCG on glycogen synthesis, the glycogen content in HepG2 cells and hepatocytes pretreated with $\mathrm{HG}(30 \mathrm{mM})$ was determined. EGCG was added to the cell cultures at concentrations of 0.01-10 $\mu \mathrm{M}$. Following stimulation of cells with $100 \mathrm{nM}$ insulin, glycogen synthesis decreased by $~ 50 \%$ in HepG 2 cells $(\mathrm{P}<0.01)$ and primary hepatocytes $(\mathrm{P}<0.01)$ exposed to $\mathrm{HG}$ when compared to those treated with $100 \mathrm{nM}$ insulin plus LG, indicating that $\mathrm{HG}$ treatment may lead to insulin resistance (Fig. 2). By contrast, when cells were pre-treated with EGCG (0.01-10 $\mu \mathrm{M})$, glycogen synthesis was gradually restored in HepG2 cells and primary hepatocytes (Fig. 2). Treatment of cells with $10 \mu \mathrm{M}$ EGCG resulted in a two-fold increase in glycogen levels in HepG2 $(\mathrm{P}<0.01)$ and primary hepatocytes $(\mathrm{P}<0.01)$ when compared with HG-only treated cells (Fig. 2). These results indicated that glycogen synthesis was improved by EGCG in a dose-dependent manner.

ROS are involved in $H G$-induced insulin resistance. To determine whether HG treatment induced ROS production in cultured HepG2 cells, ROS levels were measured using DHE staining. Following $24 \mathrm{~h}$ incubation with LG and HG, $10 \mu \mathrm{M}$ EGCG was added, and ROS production was determined using immunofluorescence and flow cytometry (Fig. 3A and B, respectively). The microimages in Fig. 3A demonstrate that ROS content was markedly reduced in HG + EGCG-treated cells compared with HG-only treated cells. As shown in Fig. 3B, HG treatment enhanced ROS production by $\sim$-fold relative to LG-treated cells $(\mathrm{P}<0.01)$.

EGCG alters the insulin-signaling pathway in hepatocytes treated with $H G$. Owing to the vital role the insulin-signaling pathway serves in glycogen synthesis (17), the authors investigated
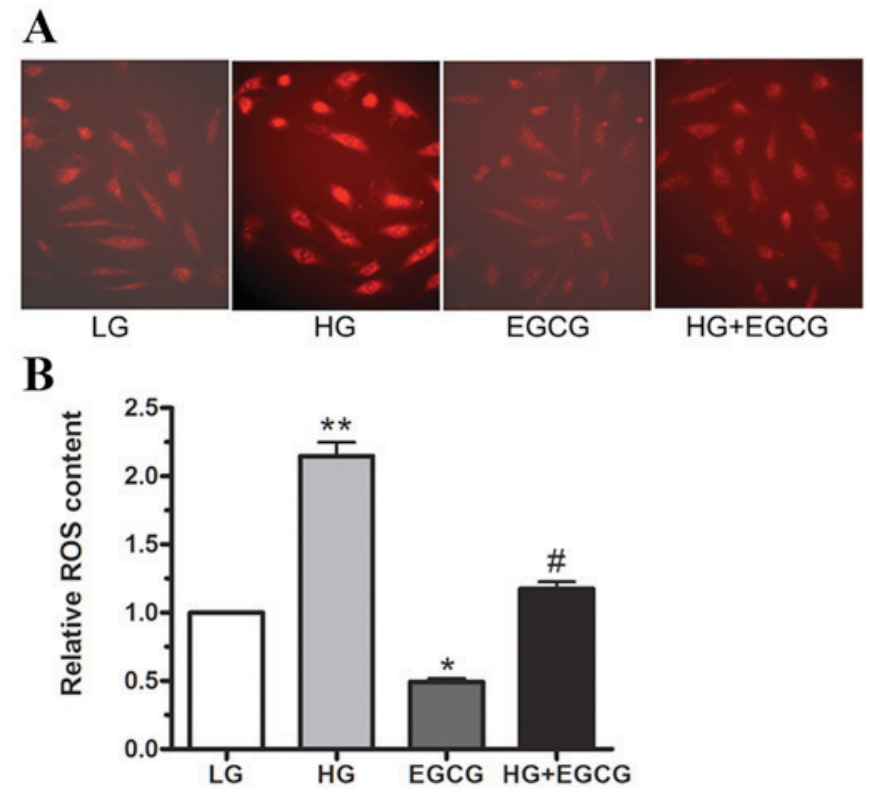

Figure 3. ROS serves an important role in HG-induced insulin resistance. (A) The effect of HG and EGCG on cellular ROS levels. HepG2 cells were stimulated with LG and HG for $24 \mathrm{~h}$ following pretreatment with $10 \mu \mathrm{M}$ EGCG. Cellular ROS levels were determined by DHE staining. Magnification, x400. (B) Flow cytometry was used to quantify ROS production in HepG2 cells treated with HG or EGCG. Data are expressed as the mean \pm standard deviation from five individual experiments. ${ }^{*} \mathrm{P}<0.05$ and ${ }^{* *} \mathrm{P}<0.01$ vs. LG; ${ }^{\#} \mathrm{P}<0.05$ vs. HG. ROS, reactive oxygen species; HG, high glucose; EGCG, epigallocatechin-3-gallate; LG, low glucose; DHE, dihydroethidium.

whether EGCG affects the insulin-signaling pathway in hepatocytes treated with EGCG. As shown in Fig. 4, a significant increase in $\mathrm{p}$-JNK expression was observed in response to HG-treatment of HepG2 cells $(\mathrm{P}<0.01)$. In addition to increased JNK phosphorylation, phosphorylation of the residue Ser307 in IRS-1 was significantly enhanced by $\mathrm{HG}$ treatment $(\mathrm{P}<0.01$; Fig. 4). HG-induced activation of JNK may have been responsible for the impaired phosphorylation of AKT and GSK (Figs. 1A and 4). However, the alterations in JNK, IRS-1, AKT and GSK expression that were induced by HG were reversed by EGCG treatment (Figs. 1 and 4). When HepG2 cells were pretreated 


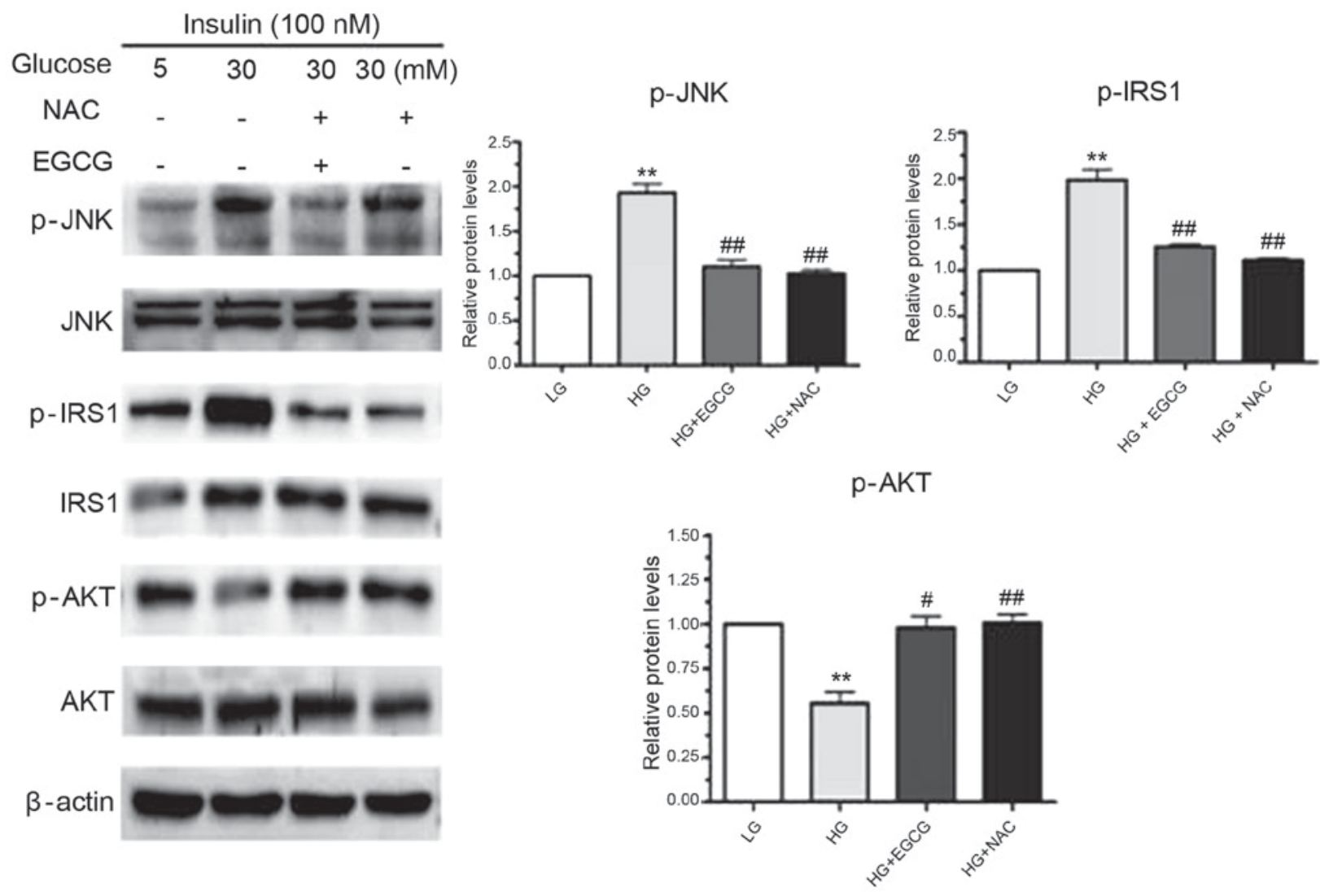

Figure 4. EGCG affects the insulin-signaling pathway in hepatocytes treated with HG. EGCG altered the JNK/IRS1/AKT insulin-signaling pathway in HepG2 cells treated with 5 or $30 \mathrm{mM} \mathrm{HG}$ for $24 \mathrm{~h}$. Data were obtained from five individual experiments and are expressed as the mean \pm standard deviation. ${ }^{* * *} \mathrm{P}<0.01$ vs. LG; ${ }^{\#} \mathrm{P}<0.05$ and ${ }^{\# \#} \mathrm{P}<0.01$ vs. HG. EGCG, epigallocatechin-3-gallate; HG, high glucose; LG, low glucose; p-JNK, phosphorylated c-JUN N-terminal kinase; IRS1, insulin receptor substrate 1; AKT, protein kinase B; GSK, glycogen synthase kinase; NAC, N-acetyl-cysteine.

with NAC and exposed to HG, JNK activation and phosphorylation of the Ser307 residue of IRS-1 were significantly reduced in NAC-treated cells compared with HG-only treated cells (p-JNK, $\mathrm{P}<0.01 ; \mathrm{p}-\mathrm{IRS}-1, \mathrm{P}<0.01 ;$ Fig. 4). These results suggested that EGCG ameliorates HG-induced insulin resistance in signaling hepatocytes by altering the ROS-induced JNK/IRS1/AKT/GSK signaling pathway.

\section{Discussion}

Type II diabetes mellitus has increasingly become a worldwide public health problem as it often leads to severe complications, including coronary disease, heart failure, retinopathy, peripheral neuropathy and hypertension $(4,18)$. Owing to the impaired capacity to utilize insulin in target organs, insulin resistance is recognized as a pathophysiological marker for type II diabetes. As an important organ in glucose metabolism, the liver serves a vital role in regulating metabolic processes. During insulin resistance, hepatic glycogen synthesis is markedly reduced and insulin signaling is impaired; these events may lead to the development of hyperglycemia and type II diabetes (19). In addition to genetic factors, insulin resistance is predominantly caused by environmental factors, including obesity, a sedentary lifestyle, pregnancy and the excess hormone production (19). Hyperglycemia is recognized as a common pathogenic factor involved in a series of additional complications in patients with type II diabetes. Previous research has indicated that increased levels of free fatty acids and glucose content inpatients with type II diabetes is associated with enhanced ROS production and oxidative stress (20). In addition, ROS has been demonstrated to severely impair the insulin-signaling pathway, which promotes the progression of type II diabetes further. Oxidative stress may lead to further tissue damage, as it is often the result of an imbalance between ROS production and antioxidant defenses (21).

At present, antidiabetic agents, such as $\alpha$-glucosidase inhibitors, amylin analogs, antidiabetic combinations, dipeptidyl peptidase 4 inhibitors, incretin mimetics and insulin, are widely prescribed for patients with type II diabetes. However, clinicians and researchers are becoming increasingly concerned with the resulting liver injury. Natural remedies extracted from medicinal plants have demonstrated their effectiveness as alternative treatments of hyperglycemia $(9,22)$. In eastern Asia, green tea is a popular traditional beverage, and previous research has indicated that regular green tea consumption reduces the risk of liver disease (23). Green tea has anti-inflammatory, antioxidative, antimutagenic and anticarcinogenic properties, which make it highly beneficial for public health (15). Furthermore, a previous epidemiological study demonstrated that regular green tea consumption may reduce the risk of developing type II diabetes (24). In streptozocin-induced diabetic mice, green tea was reported to improve hyperglycemia (22). Furthermore, green tea extracts have been demonstrated to function as effective free radical scavengers (5). EGCG is the primary polyphenol extracted from green tea and has been reported to increase fecal cholesterol excretion in rats fed on a high-fat diet when compared to controls (25). Due to the anti-hyperglycemic 
and antioxidant properties of green tea, the aim of the present study was to explore the hepatoprotective effects of EGCG in a HG-induced insulin resistance cell model.

To examine the effect of $\mathrm{HG}$ on insulin signaling, $30 \mathrm{mM}$ glucose was applied to stimulate HepG2 cells and primary hepatocytes. Compared with the LG group, HG significantly reduced the phosphorylation of AKT and GSK in the two cell types. These results indicated that HG induced insulin resistance in HepG2 and primary mouse hepatocytes. In addition, when these cells were pretreated with EGCG, the phosphorylation levels of AKT and GSK were restored, indicating the protective effects of EGCG against insulin resistance. Glycogen levels were measured using a glycogen assay kit. The results demonstrated that EGCG significantly restored glycogen synthesis in the two cell types when treated with HG. These in vitro experiments indicated that EGCG may protect hepatocytes from HG-induced insulin resistance.

Hyperglycemia is associated with the development of vascular and neurological complications, and has been implicated in their etiologies $(26,27)$. Previous studies have demonstrated that ROS production serves a major role in insulin resistance $(28,29)$. In addition, in vitro studies have revealed that enhanced ROS production activates multiple serine kinase cascades (30). In the insulin-signaling pathway, the insulin receptor and IRS are potential targets of these activated kinases $(31,32)$. It has been reported that enhanced IRS serine phosphorylation reduced the level of tyrosine phosphorylation, thereby decreasing insulin activity (33). Furthermore, it has been demonstrated that ROS enhances JNK serine phosphorylation, which increases the serine phosphorylation of the IRS protein (34). Enhanced IRS-1 serine phosphorylation impairs downstream insulin signaling. AKT and GSK activation is subsequently reduced, followed by the reduction of glycogen synthase activity (35).

In the present study, HepG2 cells were pre-treated with $30 \mathrm{mM}$ glucose and cellular ROS levels were determined by DHE staining and flow cytometry. The results indicated that HG treatment significantly enhanced ROS production when compared with the LG-treated cells. In addition, HG plus EGCG treatment, led to a significant reduction in ROS production when compared with HG-only treated cells. The results suggest that HG may increase ROS content in patients with insulin resistance. EGCG was observed to protect the cells from abnormal ROS production. To further explore the protective effect of EGCG on insulin resistance, the expression levels of key proteins associated with insulin signaling were analyzed. The results revealed that, in addition to the increased level of JNK phosphorylation, phosphorylation of the residue Ser307 in IRS-1 was enhanced in HepG2 cells treated with HG. In addition, HG-induced activation of JNK may have led to impaired phosphorylation of AKT and GSK. However, the HG-induced alterations to JNK, IRS-1, AKT and GSK expression were reversed by EGCG treatment. To further validate the protective effects of EGCG, NAC (a well-known antioxidant) was applied. When HepG2 cells were pre-treated with NAC and HG for $1 \mathrm{~h}$, serine phosphorylation of JNK and IRS1 was significantly decreased in both EGCG and NAC-treated cells. This was similar to the effect of EGCG exposure. Thus, EGCG improved insulin signaling potentially through the reduction of ROS production in hepatocytes.

In conclusion, the results of the present study demonstrated that HG may induce hepatic insulin resistance. In addition, ROS may serve a major role in the pathology of insulin resistance through JNK and IRS1 serine phosphorylation. Furthermore, EGCG decreased ROS production and affected the insulin-signaling pathway. Therefore, green tea extracts may be a promising therapeutic intervention for insulin resistance in patients with type II diabetes.

\section{References}

1. Ezenwaka CE, Okoye O, Esonwune C, Onuoha P, Dioka C, Osuji C, Oguejiofor C and Meludu S: High prevalence of abdominal obesity increases the risk of the metabolic syndrome in Nigerian type 2 diabetes patients: Using the International diabetes federation worldwide definition. Metab Syndr Relat Disord 12: 277-282, 2014.

2. Cable JC, Tan GD, Alexander SP and O'Sullivan SE: The effects of obesity, diabetes and metabolic syndrome on the hydrolytic enzymes of the endocannabinoid system in animal and human adipocytes. Lipids Health Dis 13: 43, 2014.

3. Liu CY, Huang CJ, Huang LH, Chen IJ, Chiu JP and Hsu CH: Effects of green tea extract on insulin resistance and glucagon-like peptide 1 in patients with type 2 diabetes and lipid abnormalities: A randomized, double-blinded, and placebo-controlled trial. PLoS One 9: e91163, 2014.

4. Gilbert RE: The endothelium in diabetic nephropathy. Curr Atheroscler Rep 16: 410, 2014.

5. Crespy V and Williamson G: A review of the health effects of green tea catechins in in vivo animal models. J Nutr 134 (Suppl 12): S3431-S3440, 2004.

6. Basu A, Sanchez K, Leyva MJ, Wu M, Betts NM, Aston CE and Lyons TJ: Green tea supplementation affects body weight, lipids, and lipid peroxidation in obese subjects with metabolic syndrome. J Am Coll Nutr 29: 31-40, 2010.

7. Sae-Tan S, Grove KA and Lambert JD: Weight control and prevention of metabolic syndrome by green tea. Pharmacol Res 64: 146-154, 2011.

8. Ihm SH, Jang SW, Kim OR, Chang K, Oak MH, Lee JO, Lim DY and Kim JH: Decaffeinated green tea extract improves hypertension and insulin resistance in a rat model of metabolic syndrome. Atherosclerosis 224: 377-383, 2012.

9. Sae-Tan S, Rogers CJ and Lambert JD: Voluntary exercise and green tea enhance the expression of genes related to energy utilization and attenuate metabolic syndrome in high fat fed mice. Mol Nutr Food Res 58: 1156-1159, 2014.

10. Thielecke F and Boschmann M: The potential role of green tea catechins in the prevention of the metabolic syndrome-A review. Phytochemistry 70: 11-24, 2009.

11. Vieira Senger AE, Schwanke CH, Gomes I and Valle Gottlieb MG: Effect of green tea (Camellia sinensis) consumption on the components of metabolic syndrome in elderly. J Nutr Health Aging 16: 738-742, 2012

12. Cia D, Vergnaud-Gauduchon J, Jacquemot $\mathrm{N}$ and Doly M: Epigallocatechin gallate (EGCG) prevents $\mathrm{H} 2 \mathrm{O} 2$-induced oxidative stress in primary rat retinal pigment epithelial cells. Curr Eye Res 39: 944-952, 2014.

13. Yang EJ, Lee J, Lee SY, Kim EK, Moon YM, Jung YO, Park SH and Cho ML: EGCG attenuates autoimmune arthritis by inhibition of STAT3 and HIF-1 $\alpha$ with Th17/Treg control. PLoS One 9: e86062, 2014.

14. Zhou J, Farah BL, Sinha RA, Wu Y, Singh BK, Bay BH, Yang CS and Yen PM: Epigallocatechin-3-gallate (EGCG), a green tea polyphenol, stimulates hepatic autophagy and lipid clearance. PLoS One 9: e87161, 2014.

15. Benelli R, Venè R, Bisacchi D, Garbisa S and Albini A: Anti-invasive effects of green tea polyphenol epigallocatechin-3-gallate (EGCG), a natural inhibitor of metallo and serine proteases. Biol Chem 383: 101-105, 2002.

16. Zhao C, She T, Wang L, Su Y, Qu L, Gao Y, Xu S, Cai S and Shou C: Daucosterol inhibits cancer cell proliferation by inducing autophagy through reactive oxygen species-dependent manner. Life Sci 137: 37-43, 2015.

17. Saltiel AR: Insulin signaling in the control of glucose and lipid homeostasis. Handb Exp Pharmacol 233: 51-71, 2016.

18. Hendriksen PH, Oey PL, Wieneke GH, Bravenboer B and Banga JD: Subclinical diabetic neuropathy: Similarities between electrophysiological results of patients with type 1 (insulin-dependent) and type 2 (non-insulin-dependent) diabetes mellitus. Diabetologia 35: 690-695, 1992. 
19. Eddouks M, Maghrani $M$ and Michel JB: Hypoglycaemic effect of Triticum repens P. Beauv. in normal and diabetic rats. J Ethnopharmacol 102: 228-232, 2005.

20. Stefano GB, Challenger S and Kream RM: Hyperglycemia-associated alterations in cellular signaling and dysregulated mitochondrial bioenergetics in human metabolic disorders. Eur J Nutr 55: 2339-2345, 2016.

21. Bukhari SA, Naqvi SA, Nagra SA, Anjum F, Javed S and Farooq M: Assessing of oxidative stress related parameters in diabetes mellitus type 2: Cause excessive damaging to DNA and enhanced homocysteine in diabetic patients. Pak J Pharm Sci 28: 483-491, 2015.

22. Tsuneki H, Ishizuka M, Terasawa M, Wu JB, Sasaoka T and Kimura I: Effect of green tea on blood glucose levels and serum proteomic patterns in diabetic $(\mathrm{db} / \mathrm{db})$ mice and on glucose metabolism in healthy humans. BMC Pharmacol 4: 18, 2004.

23. Hirsch N, Konstantinov A, Anavi S, Aronis A, Hagay Z, Madar Z and Tirosh O: Prolonged feeding with green tea polyphenols exacerbates cholesterol-induced fatty liver disease in mice. Mol Nutr Food Res 60: 2542-2553, 2016.

24. Weisburger JH and Chung FL: Mechanisms of chronic disease causation by nutritional factors and tobacco products and their prevention by tea polyphenols. Food Chem Toxicol 40: 1145-1154, 2002.

25. Raederstorff DG, Schlachter MF, Elste V and Weber P: Effect of EGCG on lipid absorption and plasma lipid levels in rats. J Nutr Biochem 14: 326-332, 2003.

26. Rösen P, Nawroth PP, King G, Moller W, Tritschler HJ and Packer L: The role of oxidative stress in the onset and progression of diabetes and its complications: A summary of a congress series sponsored by UNESCO-MCBN, the American diabetes association and the German diabetes society. Diabetes Metab Res Rev 17: 189-212, 2001.
27. Nishikawa T, Edelstein D and Brownlee M: The missing link: A single unifying mechanism for diabetic complications. Kidney Int Suppl 77: S26-S30, 2000.

28. Paolisso $\mathrm{G}$ and Giugliano D: Oxidative stress and insulin action: Is there a relationship? Diabetologia 39: 357-363, 1996.

29. Rudich A, Kozlovsky N, Potashnik R and Bashan N: Oxidant stress reduces insulin responsiveness in 3T3-L1 adipocytes. Am J Physiol 272: E935-E940, 1997.

30. Kyriakis JM and Avruch J: Sounding the alarm: Protein kinase cascades activated by stress and inflammation. J Biol Chem 271: 24313-24316, 1996.

31. Blair AS, Hajduch E, Litherland GJ and Hundal HS: Regulation of glucose transport and glycogen synthesis in L6 muscle cells during oxidative stress. Evidence for cross-talk between the insulin and SAPK2/p38 mitogen-activated protein kinase signaling pathways. J Biol Chem 274: 36293-36299, 1999.

32. Birnbaum MJ: Turning down insulin signaling. J Clin Invest 108: 655-659, 2001

33. Liu K, Zhao W, Gao X, Huang F, Kou J and Liu B: Diosgenin ameliorates palmitate-induced endothelial dysfunction and insulin resistance via blocking IKK $\beta$ and IRS-1 pathways. Atherosclerosis 223: 350-358, 2012.

34. Katakam AK, Chipitsyna G, Gong Q, Vancha AR, Gabbeta J and Arafat HA: Streptozotocin (STZ) mediates acute upregulation of serum and pancreatic osteopontin (OPN): A novel islet-protective effect of OPN through inhibition of STZ-induced nitric oxide production. J Endocrinol 187: 237-247, 2005.

35. Chu J, Zhang H, Huang X, Lin Y, Shen T, Chen B, Man Y, Wang S and Li J: Apelin ameliorates TNF- $\alpha$-induced reduction of glycogen synthesis in the hepatocytes through $\mathrm{G}$ protein-coupled receptor APJ. PLoS One 8: e57231, 2013. 